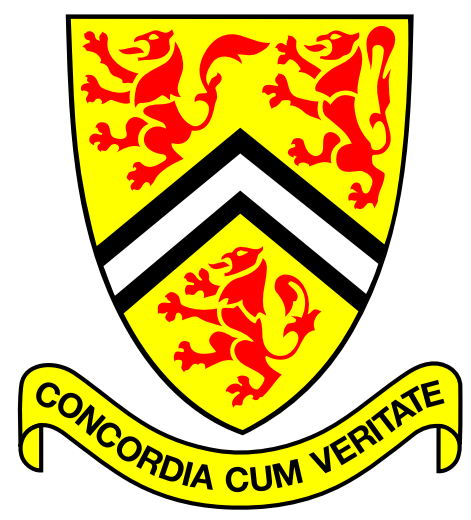

A New Non-Orthogonal Space-Time Code with Low Decoding Complexity

Mohammad Ali Maddah-Ali and Amir K. Khandani

Coding \& Signal Transmission Laboratory

Department of Electrical \& Computer Engineering

University of Waterloo

Waterloo, Ontario, Canada, N2L 3G1

Technical Report UW-E\&CE 2004-04

April 1, 2004 


\title{
A New Non-Orthogonal Space-Time Code with Low Decoding Complexity
}

\author{
Mohammad Ali Maddah-Ali and Amir K. Khandani \\ Coding \& Signal Transmission Laboratory(www.cst.uwaterloo.ca) \\ Dept. of Elec. and Comp. Eng., University of Waterloo \\ Waterloo, ON, Canada, N2L 3G1
}

Tel: 519-8833950, Fax: 519-8884338

e-mail: \{mohammad, khandani\}@cst.uwaterloo.ca

\begin{abstract}
A full diversity block space-time code over two transmit antennas and two symbol periods is introduced. In this method, each code is equal to the addition of two matrices; $\mathbf{A}^{m}$ and $\mathbf{D} \mathbf{A}^{n}$, where $\mathbf{A}$ and $\mathbf{D}$ are the two constant matrices and $m$ and $n$ are the two data symbols. $\mathbf{A}$ is selected such that the set $\mathbf{A}^{m}, 0 \leq m \leq 2^{b}-1$ is closed under the matrix multiplication. This structure allows a simple Maximum Likelihood (ML) decoding method, and at the same time, simplifies the optimization of the coding advantage. Simulations show that the performance of the new code is very close to that of the Damen code [1] which is the best known block space-time code in terms of the coding advantage. Moreover, the decoding complexity of the proposed method is significantly lower than that of the Damen code.
\end{abstract}

\section{INTRODUCTION}

$\mathbf{R}$ ECENTLY, the use of multiple antennas at both ends of wireless links has received signifi cant attention in order to increase the communication rate and/or to exploit the diversity [2]-[4]. Space-time code, a combination of modulation and channel coding across the space and the time dimensions, has been introduced by Tarokh et al. to take advantage of the spatial diversity [4]. Authors have introduced two criteria, based on the rank and the determinant of the substraction of the code matrices, to design space-time 
codes. These design criteria have been successfully used in other research [5]-[9]. Later, other design criteria have been developed for special scenarios such as low Signal to Noise Ratio (SNR), high data rates, and a large number of antennas [10]-[14].

Block orthogonal space-time coding is another method of designing full diversity space-time codes with an orthogonality property which simplifi es decoding [5] [15]. However, it has been demonstrated that the use of an orthogonal structure sacrifi ces the achievable coding gain. Moreover, this structure is restrictive in the sense that it does not allow to achieve the channel capacity for more than one receive antenna [14]. A well known example of the orthogonal code is the so-called Alamouti code [15] which takes advantage of two transmit antennas over two symbol periods.

Knowing the sub-optimality of the Alamouti code and considering its practical signifi cance, researchers have attempted to improve this code. For example, some have focused on improving the coding advantage of the orthogonal Space-Time Block Code (STBC) by concatenating the Alamouti scheme with a forward error correcting code [16][19]. Also, there have been some attempts to construct a code with a high rate by relaxing the orthogonality. In [20], a quasi-orthogonal code, based on using the Alamouti code as the building block is introduced. The rate of this code is one symbol Per Channel Use (PCU), however it offers a diversity which is only half of the maximum possible value. In [21], a full rate code is proposed to minimize a defi ned measure of the non-orthogonality. Non-orthogonality arises from increasing the rate above the maximum that is possible with an orthogonal structure.

There have been some attempts to increase the coding advantage by relaxing the orthogonality. Hughes in [22], [23] has generalized the concept of group codes for multiple antenna systems. This class of codes is fully diverse and has distance invariance property. However, the rate of the code cannot achieve the maximum possible value (for full diversity, the maximum achieveable rate is one symbol PCU [4].) The best known work in the category of non-orthogonal codes is [1] [8]. Here, an algebraic structure is utilized to construct non-orthogonal codes with a large coding advantage. For decoding, the authors suggest the sphere decoding algorithm (see [24]) that is generally a complex 
operation. However, fi nding codes with good distance properties which at the same time allow the use of a simple Maximum Likelihood (ML) decoding algorithm remains an open problem.

In this paper, a new full-diversity full-rate space-time code is introduced. In this method, each code is equal to the addition of two matrices; $\mathbf{A}^{m}$ and $\mathbf{D} \mathbf{A}^{n}$, where $m$ and $n$ are two data symbols and $\mathbf{A}$ and $\mathbf{D}$ are two constant matrices. $\mathbf{A}$ is selected such that the set $\mathbf{A}^{m}, 0 \leq m \leq 2^{b}-1$ is closed under matrix multiplication. This structure allows a simple Maximum Likelihood (ML) decoding method, and at the same time, simplifi es the optimization of the coding advantage. This code is over two transmit antennas which has a signifi cant practical importance and has been the subject of numerous papers [1], [15], [25]-[27]. Simulations show that the performance of the new code is very close to that of the Damen code [1] which is the best known block space-time code in terms of the coding advantage. Moreover, the decoding complexity of the proposed method is signifi cantly less than that of the Damen code.

The rest of this paper is organized as follows: In Section II, the system model and the basic design criteria are introduced. The structure of the proposed code is presented in Section III. In addition, a method to optimize the coding advantage is explained. In Section IV, a novel algorithm for the decoding of the proposed method is developed. In Section $\mathrm{V}$, the simulation results and comparisons with the best alternative method are presented. Finally, the complexity of the proposed decoding method is compared with that of the Damen code which is based on sphere decoding.

\section{PRELIMINARIES}

In a slow flat fading environment, Multiple Input Multiple Output (MIMO) channels with $M$ transmit antennas and $N$ receive antennas over $T$ symbol periods is modeled by

$$
\mathbf{Y}=\sqrt{\frac{\rho}{M}} \mathbf{H X}+\mathbf{V},
$$

where $\mathbf{Y} \in \mathcal{C}^{N \times T}$ denotes the received matrix, $\mathbf{X} \in \mathcal{C}^{M \times T}$ represents the transmitted matrix, $\mathbf{H} \in \mathcal{C}^{N \times M}$ signifi es the channel matrix, and $\mathbf{V} \in \mathcal{C}^{N \times T}$ is the additive, spatially 
and temporally i.i.d white noise with a zero-mean unit-variance complex Gaussian distribution. Matrix X, selected from a fi nite set, the codebook, and noise $\mathbf{V}$ are normalized such that $\rho$ is the SNR at each receive antenna. Considering the two matrices $\mathbf{X}_{\eta}$ and $\mathbf{X}_{\mu}$ in the codebook, we defi ne $\mathbf{B}_{\eta \mu}$ as $\mathbf{B}_{\eta \mu}=\left(\mathbf{X}_{\eta}-\mathbf{X}_{\mu}\right)\left(\mathbf{X}_{\eta}-\mathbf{X}_{\mu}\right)^{*}$, where $\left(\mathbf{X}_{\eta}-\mathbf{X}_{\mu}\right)^{*}$ is the conjugate transpose of the matrix $\left(\mathbf{X}_{\eta}-\mathbf{X}_{\mu}\right)$. The following are the criteria for space-time code design [4]:

- Rank Criterion: The diversity advantage is defi ned as the minimum rank of the matrices $\mathbf{B}_{\eta \mu}$ over all the pairs of codewords in the codebook. In order to achieve full diversity, the matrices $\mathbf{B}_{\eta \mu}$ have to be full rank for all pairs in the codebook.

- Determinant Criterion: The coding advantage is defi ned as the minmum of $\left[\prod_{l=1}^{\tau} \lambda_{l}\left(\mathbf{B}_{\eta \mu}\right)\right]^{1 / \tau}$, where $\lambda_{l}\left(\mathbf{B}_{\eta \mu}\right), l=1 \cdots \tau$ are the nonzero eigenvalues of $\mathbf{B}_{\eta \mu}$. For a fully diverse code, the coding advantage is equal to the minimum of $\left[\operatorname{det}\left(\mathbf{B}_{\eta \mu}\right)\right]^{1 / \tau}$.

\section{CODE STRUCTURE}

In this section, we introduce a new full-rate full-diversity block space-time code with a simple decoding method and high coding advantage.

The proposed structure is based on the PSK constellation with $2^{b}$ points. The codebook is composed of the following elements:

$$
C=\left\{\mathbf{C}(m, n)=\mathbf{C}(m, n)=\mathbf{A}^{m}+\mathbf{D A}^{n}, \quad \text { for } \quad m, n=0,1, \ldots, 2^{b}-1\right\}
$$

where $m$ and $n$ denote two data symbols,

$$
\mathbf{A}=\left[\begin{array}{cc}
\theta_{1} & 0 \\
0 & \theta_{r}
\end{array}\right] \quad \mathbf{D}=\left[\begin{array}{cc}
0 & \theta_{s} \\
1 & 0
\end{array}\right]
$$

and

$$
\theta_{n}=\exp \left(j \frac{2 \pi}{2^{b}} n\right)
$$

Parameters $r$ and $s$ are selected for optimizing the coding advantage while maintaining the maximum diversity. The throughput of the code is equal to $b$ bits or one symbol per channel use, the maximum achievable rate for a fully divere code. 
According to (2), each codeword $\mathbf{C}(m, n)$ is equal to the addition of two matrices namely $\mathbf{A}^{m}$ and $\mathbf{D A}{ }^{n}$; each of them just depends on one of the data symbols $m$ or $n$. In addition, it is evident that $\mathbf{A}^{-1}=\mathbf{A}^{*}$ and $\mathbf{A}^{2^{b}}=\mathbf{I}$, where $\mathbf{I}$ is the identity matrix. In the next section, we show that this structure simplifies the Maximum Likelihood (ML) decoding. It also allows the use of a simple procedure for optimizing the coding advantage, as explained in the following.

Consider the following codebook:

$$
C=\left\{\mathbf{C}(m, n) \in \mathcal{C}^{2 \times 2} \quad m, n=0,1, \ldots, 2^{b}-1\right\} .
$$

The main objective is to maximize the coding advantage which is defi ned by the following:

$$
\Delta=\min _{m, n, \widehat{m}, \widehat{n}}\left\{\operatorname{det}\left([\mathbf{C}(m, n)-\mathbf{C}(\widehat{m}, \widehat{n})][\mathbf{C}(m, n)-\mathbf{C}(\widehat{m}, \widehat{n})]^{*}\right)\right\}^{1 / 2}
$$

for all the different pairs of $(m, n)$ and $(\widehat{m}, \widehat{n})$.

Using the basic matrix theory, we can rewrite the coding advantage as follows:

$$
\Delta=\min _{m, n, \widehat{m}, \widehat{n}}|\operatorname{det}[\mathbf{C}(m, n)-\mathbf{C}(\widehat{m}, \widehat{n})]|,
$$

where $|$.$| denotes the magnitude of a complex number.$

According to (2),

$$
\operatorname{det}[\mathbf{C}(m, n)-\mathbf{C}(\widehat{m}, \widehat{n})]=\operatorname{det}\left(\mathbf{A}^{m}+\mathbf{D} \mathbf{A}^{n}-\mathbf{A}^{\widehat{m}}-\mathbf{D} \mathbf{A}^{\widehat{n}}\right) .
$$

Since $\mathbf{C}(m, n)-\mathbf{C}(\widehat{m}, \widehat{n})$ is a $2 \times 2$ matrix and since $\mathbf{A}^{m}-\mathbf{A}^{\widehat{m}}$ is diagonal and also the main diagonal entries of $\mathbf{D}\left(\mathbf{A}^{m}-\mathbf{A}^{\widehat{m}}\right)$ are zero, it is obvious that

$$
\operatorname{det}[\mathbf{C}(m, n)-\mathbf{C}(\widehat{m}, \widehat{n})]=\operatorname{det}\left(\mathbf{A}^{m}-\mathbf{A}^{\widehat{m}}\right)+\operatorname{det}(\mathbf{D}) \operatorname{det}\left(\mathbf{A}^{n}-\mathbf{A}^{\widehat{n}}\right) .
$$

By using the vector interpretation of the complex numbers, (9) is represented on the complex plane in fig. 1 . As it is shown in this figure, the vector $\operatorname{det}[\mathbf{C}(m, n)-\mathbf{C}(\widehat{m}, \widehat{n})]$ is equal to the addition of the two vectors: $\operatorname{det}\left(\mathbf{A}^{m}-\mathbf{A}^{\widehat{m}}\right)$ and $\operatorname{det}(\mathbf{D}) \operatorname{det}\left(\mathbf{A}^{n}-\mathbf{A}^{\widehat{n}}\right)$. Note that $\operatorname{det}(\mathbf{D})=-\exp \left(s \frac{2 \pi}{2^{b}}\right)$. Therefore, $\operatorname{det}(\mathbf{D})$ is a vector with a magnitude equal 


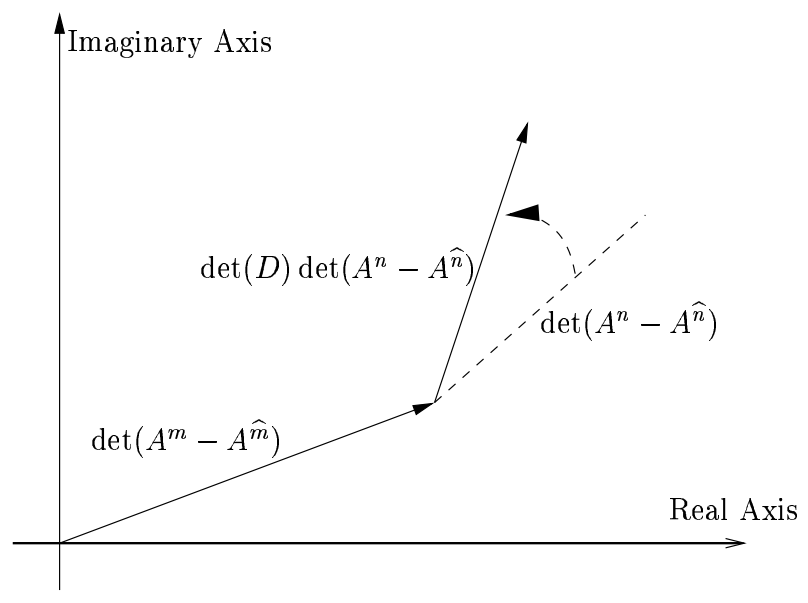

Fig. 1. Vector representation of $\operatorname{det}[\mathbf{C}(m, n)-\mathbf{C}(\widehat{m}, \widehat{n})]$

to one and is used to change the direction of the second vector to increase the minmum length of $\operatorname{det}[\mathbf{C}(m, n)-\mathbf{C}(\widehat{m}, \widehat{n})]$ which is the coding advantage.

Consequently, we can decouple the optimization procedure for selecting the parameters $r$ and $s$, resulting in the following low complexity, sub-optimum decoding procedure.

- Step One: The parameter $r$ must be chosen to maximize the minimum of the $\left|\operatorname{det}\left(\mathbf{A}^{m}-\mathbf{A}^{\widehat{m}}\right)\right|$ for all different values of $m$ and $\widehat{m}$. (or $\left|\operatorname{det}\left(\mathbf{A}^{n}-\mathbf{A}^{\widehat{n}}\right)\right|$ for all the different values of $n$ and $\widehat{n}$.)

Since $\mathbf{A}$ is a unitary matrix, $\left|\operatorname{det}\left(\mathbf{A}^{m}-\mathbf{A}^{\widehat{m}}\right)\right|=\left|\operatorname{det}\left(\mathbf{I}-\mathbf{A}^{(\widehat{m}-m)}\right)\right|$, indicating that the codebook $\left\{\mathbf{A}^{m}, m=0,1, \ldots, 2^{b}-1\right\}$ has distance invariance property. Therefore, optimizing the coding advantage is simplifi ed to finding $r$ to maximize the following function:

$$
\min _{0 \leq m \leq 2^{b}-1}\left|\mathbf{I}-\mathbf{A}^{m}\right|
$$

which is the coding advantage of the codebook $\left\{\mathbf{A}^{m}, m=0,1, \ldots, 2^{b}-1\right\}$. This optimization has been accomplished and the results are reported in [22]. It is shown that the sets $\left\{\mathbf{A}^{m}, m=0,1, \ldots, 2^{b}-1\right\}$ and $\left\{\mathbf{D A}^{n}, n=0,1, \ldots, 2^{b}-1\right\}$ are the generalized Slepian's group code [28], [22], [23]. 
- Step Two: In this step, we find the best value of $s$ (or matrix D) to maximize the coding advantage by using the selected value for $r$ in step one. The matrix $\mathbf{D}$ only changes the direction of the second vector in order to maximize the coding advantage. This rule of matrix D is mathematically explained in Appendix I. However, the best value of $s$ can be easily determined by a computer search.

In Table I, the best values for $s$ and $r$ for $2^{b} \mathrm{PSK}, 2 \leq b \leq 5$ that optimize the coding advantage are listed.

\begin{tabular}{|c|c|c|c|}
\hline & $r$ & $s$ & Coding Adv. \\
\hline \hline 4PSK & 1,3 & 1,3 & 2 \\
8PSK & 3 & $1,3,5,7$ & 1.0824 \\
16PSK & 7 & $2,6,10,14$ & 0.4483 \\
32PSK & 7,23 & $3,5,11,19,21,27,29$ & 0.1175 \\
\hline
\end{tabular}

TABLE I

OPTIMUM CODE STRUCTURE FOR $2^{b}$ PSK

\section{DECODING}

To formulate the $M L$ decoding, we have

$$
P(\mathbf{Y} \mid \mathbf{H}, \mathbf{C}(m, n))=\frac{1}{\pi^{N T}} \exp \left(-d^{2}(m, n)\right),
$$

where $\mathbf{H}$ is the channel transfer matrix, $\mathbf{Y}$ is the matrix corresponding to the received signal, and

$$
d^{2}(m, n)=\operatorname{tr}\left[\left(\mathbf{Y}-\sqrt{\frac{\rho}{M}} \mathbf{H C}(m, n)\right)\left(\mathbf{Y}-\sqrt{\frac{\rho}{M}} \mathbf{H C}(m, n)\right)^{*}\right]
$$

and "tr" denotes trace function.

The goal of the ML decoding is to fi nd $m$ and $n$ to maximize $P(\mathbf{Y} \mid \mathbf{H}, \mathbf{C}(m, n))$ or to minimize $d^{2}(m, n)$. The straight-forward approach for the ML decoding is to calculate the different values of $d^{2}(m, n)$ for all the possible values of $m$ and $n$, and to find 
the minimum value of $d^{2}(m, n)$ by using an exhaustive search. It is clear that such an exhaustive search is very complex.

Let us defi ne $K, f(n), g(m)$ and $h(m-n)$ as

$$
\begin{gathered}
K=\operatorname{tr}\left\{\mathbf{Y} \mathbf{Y}^{*}+2 \frac{\rho}{M} \mathbf{H} \mathbf{H}^{*}\right\} \\
f(m)=-\sqrt{\frac{\rho}{M}} \operatorname{tr}\left\{\mathbf{H} \mathbf{A}^{m} \mathbf{Y}^{*}+\mathbf{Y} \mathbf{A}^{-m} \mathbf{H}^{*}\right\} \\
g(n)=-\sqrt{\frac{\rho}{M}} \operatorname{tr}\left\{\mathbf{H} \mathbf{D} \mathbf{A}^{n} \mathbf{Y}^{*}+\mathbf{Y A}^{-n} \mathbf{D}^{*} \mathbf{H}^{*}\right\}
\end{gathered}
$$

and

$$
h(m-n)=\frac{\rho}{M} \operatorname{tr}\left\{\mathbf{H A}^{m-n} \mathbf{D}^{*} \mathbf{H}^{*}+\mathbf{H D A}^{n-m} \mathbf{H}^{*}\right\} .
$$

By using these notations, it is easy to show that

$$
d^{2}(m, n)=K+f(m)+g(n)+h(m-n)
$$

To prove (17), we use these facts that

$$
\mathbf{A}^{*}=\mathbf{A}^{-1} \quad \text { and } \quad \mathbf{D}^{*}=\mathbf{D}^{-1}
$$

Since $\mathbf{A}^{2^{b}}=\mathbf{A}^{0}=\mathbf{I}_{2 \times 2}, h(m-n)=h\left(m-n \bmod 2^{b}\right)$. Thus, there are $2^{b}$ different values for each of $f(m), g(n)$, and $h(m-n)$, where $0 \leq m \leq 2^{b}-1$ and $0 \leq n \leq 2^{b}-1$.

Ignoring the constant part $K$, for minimizing $f(m)+g(n)+h(m-n)$, we can use the Viterbi algorithm over the trellis structure which is exemplifi ed in Fig. 2 for the case of 8-PSK. In this fi gure, $k=[(m-n) \bmod 2]$.

Since this trellis is fully connected, it is possible to simplify the Viterbi algorithm to another effective ML decoding method with less complexity as explained by the following.

Let us sort $f(m)$ and $g(m)$ in the increasing order and specify the corresponding arguments as $m_{0}, m_{1} \ldots m_{2^{b}-1}$ and $n_{0}, n_{1} \ldots n_{2^{b}-1}$, i.e.,

$$
f\left(m_{0}\right) \leq f\left(m_{1}\right) \leq \ldots \leq f\left(m_{2^{b}-1}\right)
$$




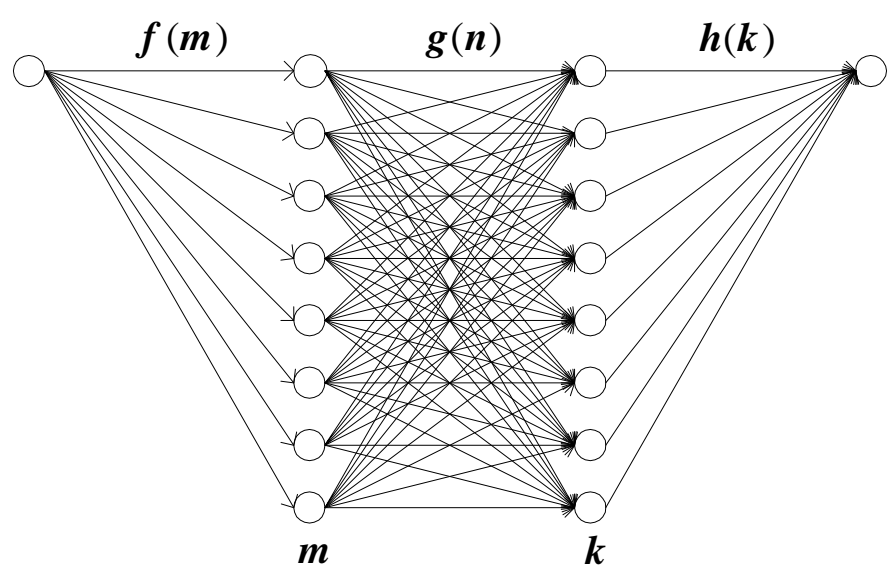

Fig. 2. Trellis construction based on code structure

and

$$
g\left(n_{0}\right) \leq g\left(n_{1}\right) \leq \ldots \leq g\left(n_{2^{b}-1}\right)
$$

We defi ne two sets, the potential set and the final set. Each set has $2^{b}$ entries corresponding to the different values of $\left[(m-n) \bmod 2^{b}\right]$. The $k^{\text {th }}$ entries of the final set $0 \leq k \leq 2^{b}-1$ is the best pair of $(m, n)$ in terms of the minimization of $f(m)+g(n)$ such that $k=\left[(m-n) \bmod 2^{b}\right]$. The $k^{\text {th }}$ entry of the potential set $0 \leq k \leq 2^{b}-1$ is the best pair of $(m, n)$ until then in terms of the minimization of $f(m)+g(n)$ such that $k=\left[(m-n) \bmod 2^{b}\right]$, and for those values of $k$ that are not yet in the final set. The final set is gradually fi lled by employing the following algorithm.

For $0 \leq \alpha \leq 2\left(2^{b}-1\right)$, starting from $\alpha=0$,

1) Find $S_{\alpha}=\left\{(p, q) \mid p+q=\alpha, 0 \leq p \leq 2^{b}-1,0 \leq q \leq 2^{b}-1\right\}$, where $p$ and $q$ are two integer numbers.

2) Find $(p, q) \in S_{\alpha}$ that minimize $f\left(m_{p}\right)+g\left(n_{q}\right)$.

3) Set $k=\left[\left(m_{p}-n_{q}\right) \bmod 2^{b}\right]$. Compare $\left(m_{p}, n_{q}\right)$ with the pair in the $k^{\text {th }}$ row of the potential set, if any, in terms of $f(m)+g(n)$, and insert the pair with the smaller value of $f(m)+g(n)$ in the $k^{\text {th }}$ row of the final set (if the $k^{\text {th }}$ row of the final set is not filled yet). 
4) For the other pairs of $(p, q) \in S_{\alpha}$, compute $k=\left[\left(m_{p}-n_{q}\right) \bmod 2^{b}\right]$ and compare $f\left(m_{p}\right)+g\left(n_{q}\right)$ with the related value in the $k^{\text {th }}$ row of the potential set. Substitute the pair with the smaller value of $f(m)+g(n)$ in the $k^{\text {th }}$ row of the potential set.

5) If the final set is not filled yet and $\alpha<2\left(2^{b}-1\right)$, set $\alpha \leftarrow \alpha+1$ and return to the first step, or else fill the empty rows of the final set with the potential set.

6) Compute $f(m)+g(n)+h(m-n)$ for the pairs in the final set and select the pair that minimizes $f(m)+g(n)+h(m-n)$.

The simulations demonstrate that the truncation of the algorithm in $\alpha=2^{b}-1$ does not considerably affect the code performance.

The idea behind the algorithm is as follows. If $(p, q) \in S_{\alpha}$ minimize $f\left(m_{p}\right)+g\left(n_{q}\right)$ and $k=\left[\left(m_{p}-n_{q}\right) \bmod 2^{b}\right]$, then there is no $\left(p^{\prime}, q^{\prime}\right) \in S_{\alpha^{\prime}},\left(\alpha<\alpha^{\prime}\right)$ such that $k=$ $\left[\left(m_{p^{\prime}}-n_{q^{\prime}}\right) \bmod 2^{b}\right]$ and $f\left(m_{p}\right)+g\left(n_{q}\right)>f\left(m_{p^{\prime}}\right)+g\left(n_{q^{\prime}}\right)$. The complete proof of the algorithm is in Appendix II.

With some modifi cations, we can use this method to decode BLAST systems (see [3]) with two transmit antennas [29].

\section{Comparison And Simulation Results}

In this section, an evaluation of the code performance by means of simulations to measure the block error rate is presented. In addition, we compare the performance of this code with that of [1]. In [1], the code is optimized for 4 bit PCU. We compare the proposed code with Damen code at this throughput. In the following comparisons, we use the optimal ML method for decoding; however, in the next section, we compare the performance of these codes considering the effects of sub-optimal decoding. For the 4-bit PCU, the 16PSK constellation is used for the modulation in the proposed code. Table II displays the coding advantage of the Damen scheme and the proposed scheme. It is apparent that the coding advantage of the novel code is very close to the coding advantage of the Damen code.

Figures 3 and 4 depict the block error rate curves for one and two receive antennas, respectively. It is apparent that the performance of the proposed code is similar to that 


\begin{tabular}{|c|c|c|}
\hline Structure & Damen Code & Proposed Code \\
\hline Coding Adv. & 0.4738 & 0.4483 \\
\hline
\end{tabular}

TABLE II

Coding Advantage of the Proposed and the Damen Codes in 4-Bits PCU

of the Damen code. In the next section, we will compare the complexity of the decoding of the proposed scheme with that of sphere decoding that is used for the decoding of the Damen code. In addition, we will compare the performances under some constraint regarding the complexity of the decoding.

\section{COMPLEXITy}

In this section, the complexity of the proposed decoding method is compared to that of the sphere decoding which is used for the Damen scheme. The maximum complexity of the proposed decoding method with a $2^{b} \mathrm{PSK}$ constellation and $N$ receiver antennas is equal to $3 \times 2^{2 b}+18 \times 2^{b}+48 N-13$ flops, where the complexity is defi ned as the total numbers of additions, multiplications, and comparisons. For instance, the maximum complexity of the decoding method for 16PSK and two receive antennas is equal to 1139 flops. Although this equation shows the maximum complexity the proposed decoding method, the simulations exhibit that the complexity for different realizations of channel matrix $\mathbf{H}$ is almost constant.

On the other hand, the complexity of sphere decoding largely varies, depending on the channel matrix $\mathbf{H}$. As a result, in order to practically implement the sphere decoding, we need to truncate the decoding algorithm whenever the complexity is greater than a pre-determined threshold. This truncation has an undesirable effect on the performance of the code. Figure 5 shows the effect of the truncation on the performance of the Damen code in the 4-bits PCU. The implementation is based on an effi cient sphere decoding that is presented in [30]. The radius of the sphere in which the search is accomplished is very important. If the radius is large, the sphere decoding will be very complex. On 
the other hand, if the radius is small, the additional error caused by decoding procedure will be very high. As a trade-off between complexity and additional error, we select the radius of the sphere such that the additional error is ten percent of the current error rate.

Figure 5 also shows that the performance of the proposed method is signifi cantly improved as compared to Damen code, if we truncate the algorithm so that the complexity is less than a given threshold. According to the curves in Fig. 5, it is easy to see that for a similar performance, we have to truncate the decoding complexity of sphere decoding to 4000 flops. Thus, when the performance is equal, the complexity of the proposed method is about four times less than the complexity of Damen code (decoded using sphere decoding.)

\section{CONCLUSION}

In this paper, a new non-orthogonal full-diversity full-rate block space-time code over two transmit antennas and two symbol periods is introduced. In this method, each code is equal to the addition of two matrices; $\mathbf{A}^{m}$ and $\mathbf{D A}{ }^{n}$, where $m$ and $n$ are the two data symbols and $\mathbf{A}$ and $\mathbf{D}$ are the two constant matrices. $\mathbf{A}$ is selected such that the set $\mathbf{A}^{m}, 0 \leq m \leq 2^{b}-1$ is closed under matrix multiplication. This structure allows a simple Maximum Likelihood (ML) decoding method, and at the same time, simplifi es the optimization of the coding advantage. A new method of decoding with a low complexity is also developed, showing about four times reduction in the decoding complexity (for similar performance) as compared to the best known alternative code introduced in [1].

\section{APPENDIX I}

AN EXPLANATION FOR DETERMINING THE PARAMETER $s$

As we mentioned in the paper, $\operatorname{det}[\mathbf{C}(m, n)-\mathbf{C}(\widehat{m}, \widehat{n})]$ has two parts: $\operatorname{det}\left(\mathbf{A}^{m}-\mathbf{A}^{\widehat{m}}\right)$ and

$\operatorname{det}(\mathbf{D}) \operatorname{det}\left(\mathbf{A}^{n}-\mathbf{A}^{\widehat{n}}\right)$. The role of the matrix $\mathbf{D}$ is to rotate the second part such that the minimum norm of the addition of this two parts is maximum. In the following, the 
selection of the matrix $\mathbf{D}$ (or parameter $s$ ) will be detailed. Let us start with following equation:

$$
\begin{aligned}
& \operatorname{det}[\mathbf{C}(m, n)-\mathbf{C}(\widehat{m}, \widehat{n})]= \\
& \operatorname{det}\left(\mathbf{A}^{m}-\mathbf{A}^{\widehat{m}}\right)+\operatorname{det}(\mathbf{D}) \operatorname{det}\left(\mathbf{A}^{n}-\mathbf{A}^{\widehat{n}}\right)= \\
& \left(e^{j m \frac{2 \pi}{2^{b}}}-e^{j \widehat{m} \frac{2 \pi}{2^{b}}}\right)\left(e^{j r m \frac{2 \pi}{2^{b}}}-e^{j r \widehat{m} \frac{2 \pi}{2^{b}}}\right)- \\
& e^{j s \frac{2 \pi}{2^{b}}}\left(e^{j n \frac{2 \pi}{2^{b}}}-e^{j \widehat{n} \frac{2 \pi}{2^{b}}}\right)\left(e^{j r n \frac{2 \pi}{2^{b}}}-e^{j r \widehat{n} \frac{2 \pi}{2^{b}}}\right)= \\
& -4 \exp \left[j(m+\widehat{m})(r+1) \frac{\pi}{2^{b}}\right] \times \sin \left[\frac{\pi}{2^{b}}(m-\widehat{m})\right] \sin \left[r \frac{\pi}{2^{b}}(m-\widehat{m})\right]- \\
& 4 \exp \left(j s \frac{2 \pi}{2^{b}}\right) \exp \left[j(n+\widehat{n})(r+1) \frac{\pi}{2^{b}}\right] \times \sin \left[\frac{\pi}{2^{b}}(n-\widehat{n})\right] \sin \left[r \frac{\pi}{2^{b}}(n-\widehat{n})\right] .
\end{aligned}
$$

Equation (21) helps us to determine the best values for $s$. In the following, the procedure for determining $s$ for 8PSK, as an example, will be explained. However, it is easy to generalize this method to a PSK constellation with a different number of points. According to $(21)$, for the 8PSK constelllation $(b=3)$, the following can be written:

$$
\begin{aligned}
& \operatorname{det}[\mathbf{C}(m, n)-\mathbf{C}(\widehat{m}, \widehat{n})]= \\
& -4 \times j^{(m+\widehat{m})} \times \sin \left[\frac{\pi}{8}(m-\widehat{m})\right] \sin \left[3 \frac{\pi}{8}(m-\widehat{m})\right]- \\
& 4 \times \exp \left(j s \frac{2 \pi}{8}\right) \times j^{(n+\widehat{n})} \sin \left[\frac{\pi}{8}(n-\widehat{n})\right] \sin \left[3 \frac{\pi}{8}(n-\widehat{n})\right] .
\end{aligned}
$$

Using the vector interpretation of complex numbers, $j^{(m+\widehat{m})} \times \sin \left[\frac{\pi}{8}(m-\widehat{m})\right] \sin \left[r \frac{\pi}{8}(m-\right.$ $\widehat{m})]$ is a vector only on the directions of the real and imaginary axes of the complex plane. The same is true for $j^{(n+\widehat{n})} \times \sin \left[\frac{\pi}{8}(n-\widehat{n})\right] \sin \left[3 \frac{\pi}{8}(n-\widehat{n})\right]$. In this case, these two vectors can cancel each other and the coding advantage will be zero. To avoid this and to maximize the coding gain, the second vector needs to be rotated. The best angles for the rotation are 45, 135, 225, and 315 degrees. Therefore, $s$ can be 1,3,5, and 7 .

\section{APPENDIX II}

\section{Proof of the Proposed Decoding Algorithm}

In this part, we prove the algorithm that is presented for decoding the proposed code. Consider $S_{\alpha}$ as defined in the first step of the algorithm. We defi ne $\left(\mathscr{A} i n(\alpha), q_{\min }(\alpha)\right)$ as follows: 


$$
\left(p_{\min }(\alpha), q_{\min }(\alpha)\right)=\arg \min _{(p, q) \in S_{\alpha}}\left\{f\left(m_{p}\right)+g\left(n_{q}\right)\right\} .
$$

It is easy to show that if $\alpha^{\prime}>\alpha$, then

$$
\exists(p, q) \in S_{\alpha} \quad \text { such that } \quad p \leq p_{\min }\left(\alpha^{\prime}\right) \quad \text { and } \quad q \leq q_{\min }\left(\alpha^{\prime}\right) .
$$

According to the inequalities (19) and (20), the inequality (24) results in

$$
\exists(p, q) \in S_{\alpha} \quad: \quad f\left(m_{p}\right)+g\left(n_{q}\right) \leq f\left(m_{p_{\min }\left(\alpha^{\prime}\right)}\right)+g\left(n_{q_{\min }\left(\alpha^{\prime}\right)}\right) .
$$

On the other hand, $(p, q) \in S_{\alpha}$; therefore based on the defi nition of $\left(p_{\min }(\alpha), q_{\min }(\alpha)\right)$,

$$
f\left(m_{p_{\min }(\alpha)}\right)+g\left(n_{q_{\min }(\alpha)}\right) \leq f\left(m_{p}\right)+g\left(n_{q}\right) .
$$

By using (25) and (26), it easy to see that,

$$
f\left(m_{p_{\text {min }}(\alpha)}\right)+g\left(n_{q_{m i n}(\alpha)}\right) \leq f\left(m_{p_{m i n}\left(\alpha^{\prime}\right)}\right)+g\left(n_{q_{m i n}\left(\alpha^{\prime}\right)}\right) .
$$

According to the defi nition of $\left(p_{\min }\left(\alpha^{\prime}\right), q_{\min }\left(\alpha^{\prime}\right)\right)$ and considering (27), we can see that:

$$
\forall(p, q) \in S_{\alpha^{\prime}} \quad: \quad f\left(m_{p_{\min }(\alpha)}\right)+g\left(n_{q_{\min }(\alpha)}\right) \leq f\left(m_{p}\right)+g\left(n_{q}\right) .
$$

So, for any $(p, q)$ in $S_{\alpha^{\prime}}$ such that $\left(m_{p_{\min }(\alpha)}-n_{q_{\min }(\alpha)} \bmod 2^{b}\right)=\left(m_{p}-n_{q}\right.$ $\left.\bmod 2^{b}\right)$,

$$
f\left(m_{p_{\min }(\alpha)}\right)+g\left(n_{q_{\min }(\alpha)}\right)+h\left(m_{p_{\min }(\alpha)}-n_{q_{\min }(\alpha)}\right) \leq f\left(m_{p}\right)+g\left(n_{q}\right)+h\left(m_{p}-n_{q}\right) .
$$

Inequality (29) guarantees that if we defi ne $k=\left(m_{p_{\text {min }}(\alpha)}-n_{q_{m i n}(\alpha)} \bmod 2^{b}\right)$, then the $k^{\text {th }}$ row of the final set should be filled with $\left(m_{p_{\min }(\alpha)}, n_{q_{\min }(\alpha)}\right)$, and there is no better choice for $(p, q)$ in $S_{\alpha^{\prime}}\left(\alpha^{\prime}>\alpha\right)$. 


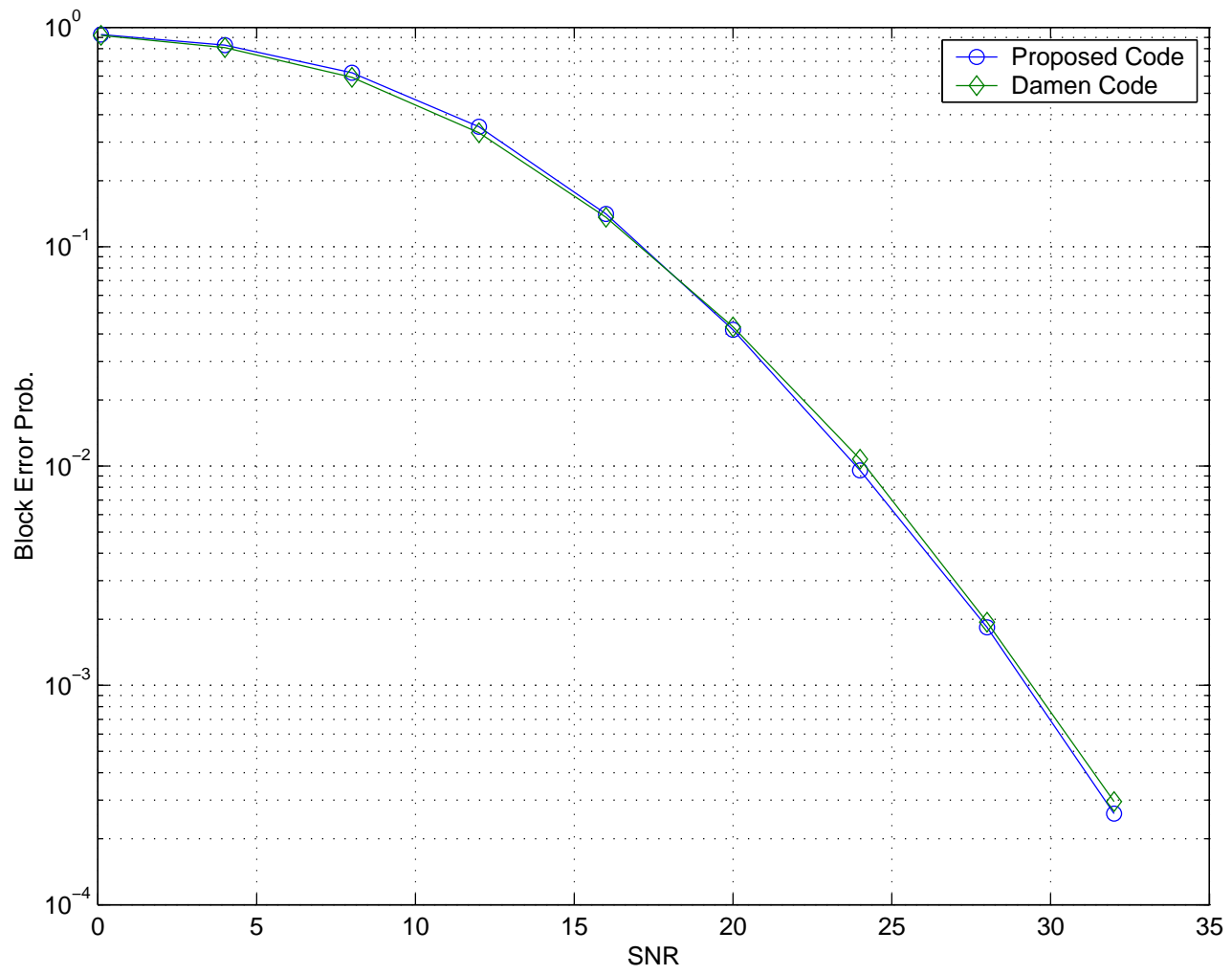

Fig. 3. Block error rate for two transmit and one receive antennas, 4-bit PCU 


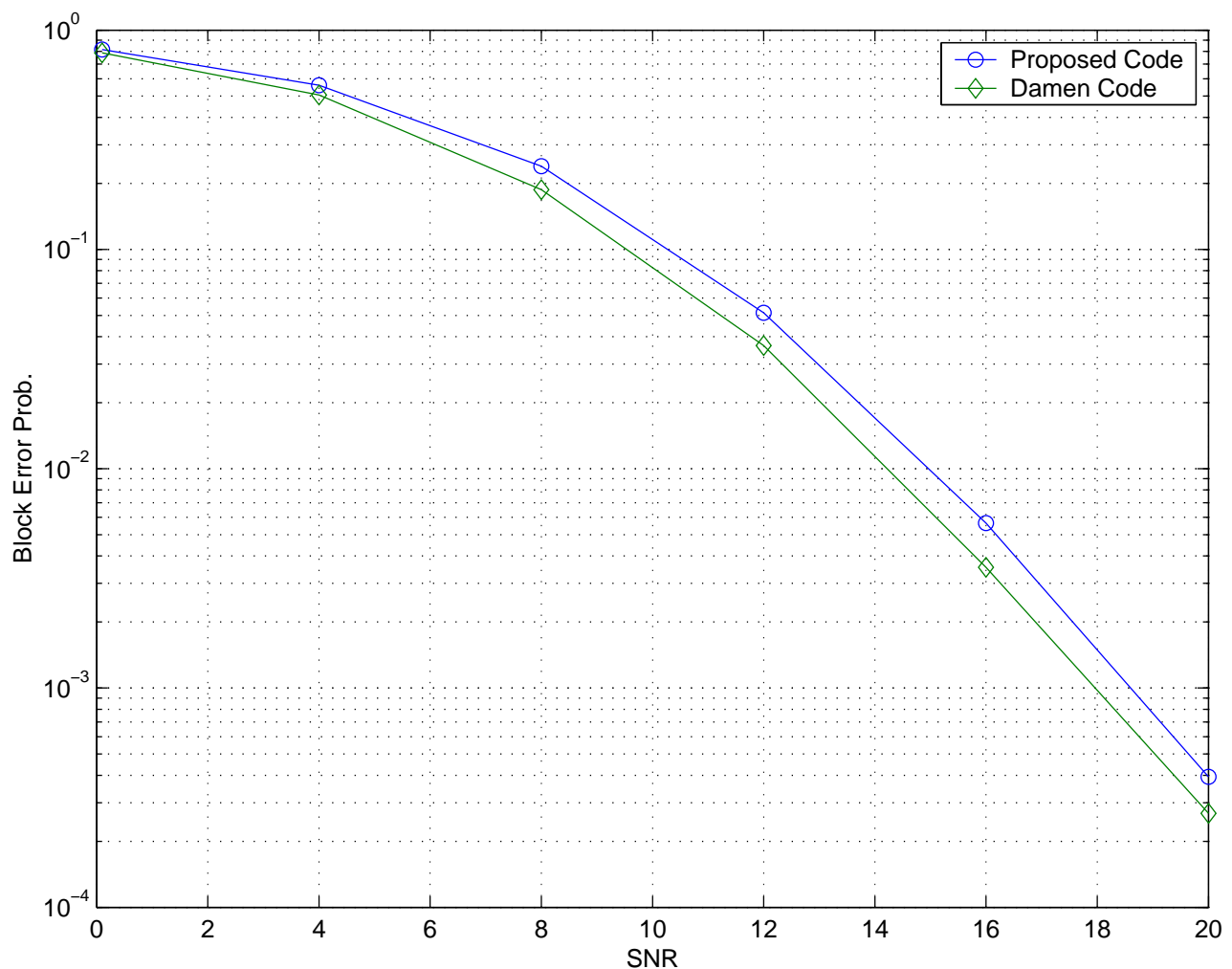

Fig. 4. Block error rate for two transmit and two receive antennas, 4-bit PCU 


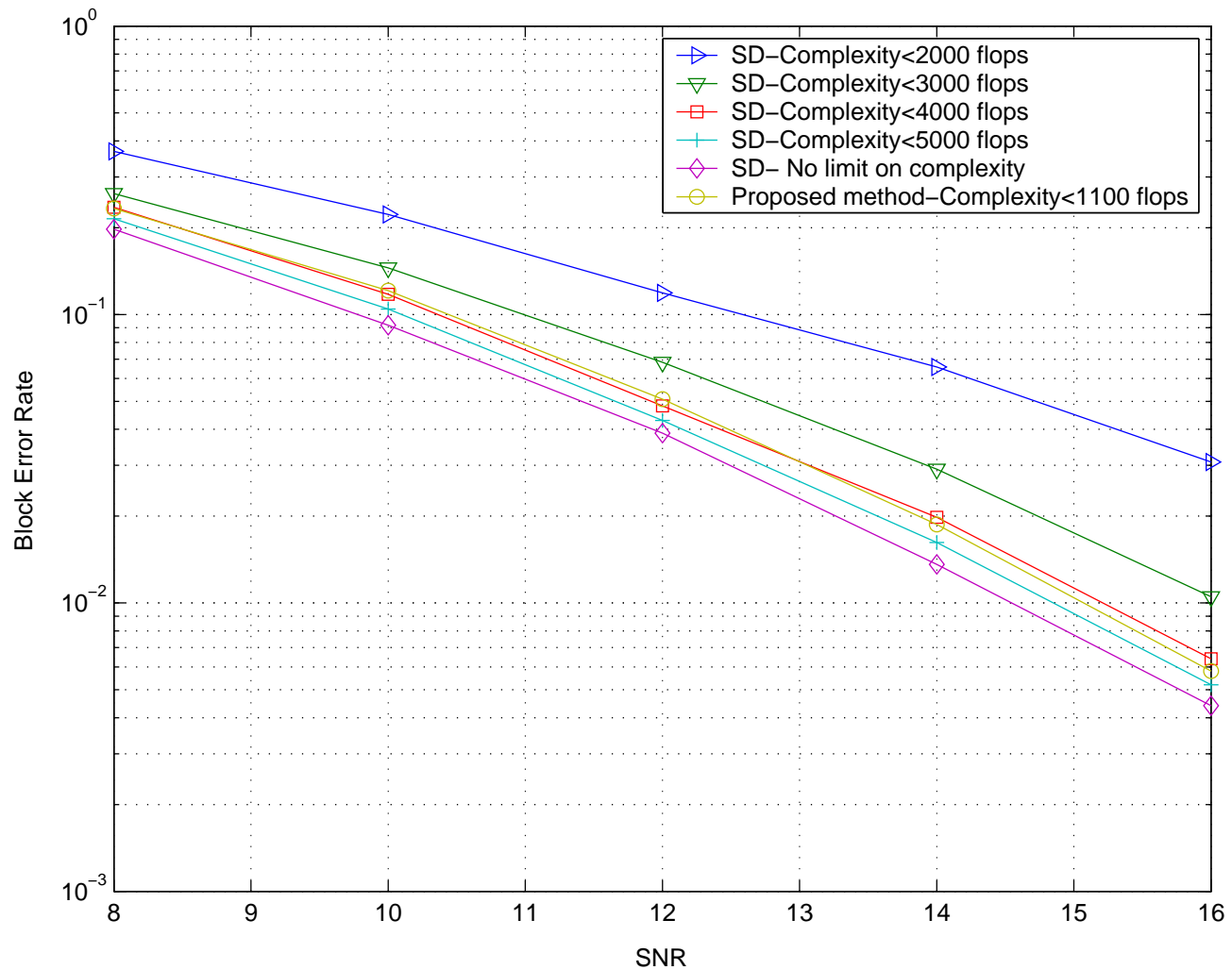

Fig. 5. Effect of the complexity truncation over Sphere Decoding(SD), as compared with the proposed method for two transmit and two reveive antennas, 4-bits PCU. 


\section{REFERENCES}

[1] M. O. Damen and A. Tewfik and J. C. Belfi ore, "A construction of space-time code based on number theory," IEEE Trans. Inform. Theory, vol. 48, pp. 753-760, Mar. 2002.

[2] I. E. Telatar, "Capacity of multi-antenna Gaussian channels," Europ. Trans. Telecommun., pp. 585-595, Nov. 1999.

[3] G. J. Foschini, "Layered space-time architecture for wireless communication in a fading environment when using multi-element antennas," Bell Labs Tech. J., pp. 41-59, Autumn 1996.

[4] V. Tarokh and N. Seshadri and A. R. Calderbank, "Space-time code for high data rate wireless communication: Performance criterion and code construction,” IEEE Trans. Inform. Theory, vol. 44, pp. 744-765, Mar. 1988.

[5] V. Tarokh and H. Jafarkhani and A. R. Calderbank, "Space-time block coding for wireless communications: Theory of generalized orthogonal designs," IEEE Trans. Inform. Theory, vol. 45, pp. 1456-1467, July 1999.

[6] H. El Gamal and M. O. Damen, "Universal space-time coding," IEEE Trans. Inform. Theory, vol. 45, pp. 1097-1119, May 2003.

[7] M. O. Damen, H. E. Gamal, and N. C. Beaulieu, "Linear threaded algebraic space-time constellations," IEEE Trans. Inform. Theory, vol. 49, pp. 2372-2388, Oct. 2003.

[8] M. O. Damen and N. C. Beaulieu, "On two high rate algebraic space-time codes," IEEE Trans. Inform. Theory, vol. 49, pp. 1059 - 1063, April 2003.

[9] H. El Gamal, A. R. Hammons, and A. Jr. Stefanov, "Space-time overlays for convolutionally coded systems," IEEE Trans. on Commun., vol. 51, pp. 1603-1612, Sept. 2003.

[10] E. Biglieri, G. Taricco, and A. Tulino, "Performance of space-time codes for a large number of antennas," IEEE Trans. Inform. Theory, vol. 48, pp. 1794 - 1803, July 2002.

[11] Z. Chen, J. Yuan, and B. Vucetic, "Improved space-time trellis coded modulation scheme on slow Rayleigh fading channels," IEE Electronics Letters, vol. 37, pp. 440-441, March 2001.

[12] M. Tao and R. S. Cheng, "Improved design criteria and new trellis codes for space-time coded modulation in slow fht fading channels," IEEE Commun. Letters, vol. 5, pp. 313-315, July 2001.

[13] D. M. Ionescu, “On space-time code design,” IEEE Trans. on Wireless Commun., vol. 2, pp. 20-28, Jan. 2003.

[14] B. Hassibi and B. M. Hochwald, "High-rate codes that are linear in space and time," IEEE Trans. Inform. Theory, vol. 48, pp. 1804-1824, July 2002.

[15] S. M. Alamouti, “A simple transmit diversity technique for wireless communication," IEEE J. Select. Areas Commun., vol. 16, pp. 1451-1458, Oct. 1998.

[16] G. Bauch, "Concatenation of space-time block codes and turbo-tcm," in IEEE International Conference on Commun., 1999, vol. 2, pp. $1202-1206$.

[17] Y. Gong and K.B. Letaief, "Concatenated space-time block coding with trellis coded modulation in fading channels,” IEEE Trans. on Wireless Commun., vol. 1, pp. 580-590, Oct. 2002.

[18] T. H. Liew and L. Hanzo, "Space-time codes and concatenated channel codes for wireless communications," Proceedings of the IEEE, vol. 90, pp. 187-219, Feb. 2002. 
[19] H. Schulze, "Performance analysis of concatenated space-time coding with two transmit antennas," IEEE Trans. on Wireless Commun., vol. 2, pp. 669-679, July 2003.

[20] H. Jafarkhani, "A quasi-orthogonal space-time block code," in IEEE Wireless Communications and Networking Conference, 2000, vol. 1, pp. 42-45.

[21] O. Tirkkonen, A. Boariu, and A. Hottinen, "Minimal non-orthogonality rate 1 space-time block code for 3+ tx antennas," in IEEE Sixth International Spread Spectrum Techniques and Applications, 2000, vol. 2, pp. 429-432.

[22] B. L. Hughes, "Differential space-time modulation," IEEE Trans. Inform. Theory, vol. 46, pp. 2567-2578, Nov. 2000.

[23] B. L. Hughes, “Optimal space-time constellation from groups,” IEEE Trans. Inform. Theory, vol. 49, pp. 401-410, Jan. 2003.

[24] U. Fincke and M. Pohst, "Improved method for calculating vectors of short length in a lattice, including a complexity analysis," Mathematics of Computation, vol. 44, April 1985.

[25] D. M. Ionescu, K. K. Mukkavilli, Z. Yan, and J. Lilleberg, "Improved 8- and 16-state space-time codes for 4psk with two transmit antennas," IEEE Commun. Letters, vol. 5, pp. 301-303, July 2003.

[26] X. B. Liang and X. G. Xia, "Unitary signal constellations for differential space-time modulation with two transmit antennas: parametric codes, optimal designs, and bounds," IEEE Trans. Inform. Theory, vol. 48, pp. 2291-2322, Aug. 2003.

[27] H. Schulze, "Performance analysis of concatenated space-time coding with two transmit antennas," IEEE Trans. on Wireless Commun., vol. 2, pp. 669-679, July 2003.

[28] D. Slepian, “Group codes for Gaussian channel,” Bell Syst. Tech. J., vol. 47, pp. 575-602, Apr. 1968.

[29] M. A. Maddah-Ali, A. K. Khandani, and W. Tong, "A new low complexity optimal decoding method for mimo systems," in In proceeding of 58th IEEE Vehicular Technology Conference, Oct. 2003.

[30] B. Hassibi and H. Vikalo, “On sphere decoding algorithm, part one: expected complexity," IEEE Trans. on Signal Processing, submitted for Publication. 